VIII ${ }^{\text {èmes }}$ Journées Nationales Génie Civil - Génie Côtier, Compiègne, 7-9 septembre 2004

\title{
Longueur de mélange et diffusion turbulente de sédiments
}

\author{
Rafik Absi et Stéphane Marchandon
}

\author{
Enseignants-chercheurs \\ Ecole de Biologie Industrielle -32, Bd du Port, 95094 Cergy-Pontoise
}

\section{Résumé}

Pour décrire le profil de concentration des sédiments en suspension dans un écoulement turbulent, nous introduisons dans cette contribution une nouvelle écriture du profil vertical $\operatorname{lm}(\mathrm{z})$ de la longueur de mélange.

Nous montrons que notre expression de $1 \mathrm{~m}$ ne tend en limite vers celle de Prandtl que dans le cas des faibles rugosités de surface (conditions aux limites du type « fond lisse »). Sous l'hypothèse de validité de la loi de Fick, le profil de concentration obtenu est qualitativement comparé aux prévisions théoriques usuelles, basées sur $1 \mathrm{~m}=\kappa \mathrm{z}$

L'écart constaté permet $d$ 'expliquer -sans hypothèse arbitraire -certains résultats expérimentaux.

Enfin, une critique est faite de 1'usage de la loi de Fick pour 1'écriture du flux de mélange dans le cas des forts gradients de concentration. Mots clés Sédiments, turbulence, diffusion, mélange, longueur, rugosité.

\section{Abstract}

In order to describe the suspended sediment concentration profile in a turbulent flow, we introduce in this contribution a new vertical profile for the mixing length $\operatorname{lm}(\mathrm{z})$.

We show that our expression tends to the Prandtl mixing length only in the case of small roughness (smooth bed boundary condition). Under the assumption of validity of the Fickian (or gradient) diffusion, the obtained concentration profile is qualitatively compared to usual theoretical predictions, based on $\mathrm{lm}=\kappa \cdot z$.

The difference can explain -without arbitrary assumption -some $\mathrm{m}$ experimental data. Finally, a critical study is done on the use of the Fickian mixing flux in the case of high concentration gradients.

Keywords: Sediments, turbulence, diffusion, mixing, length, roughness.

\section{Introduction}

Le transport sédimentaire côtier et les changements morphologiques qui l'accompagnent constituent un problème majeur pour la gestion du littoral.

L'évolution de la ligne de côte et les changements des fonds marins sont provoqués par le transport des sédiments, conséquence de l'hydrodynamique de la mer composée principalement des houles engendrées par les vents et des courants dus à la propagation des marées et aux rejets en mer des eaux fluviales.

La prévision du transport sédimentaire en zones côtières et fluviales présente un défi important pour les ingénieurs concernés par des problèmes tels que la quantification du taux de transport sédimentaire et les changements morphologiques à long terme.

Il est à noter que la quantification du taux de transport sédimentaire est importante dans les applications pratiques : conception des structures de défense côtières, planification des procédures d'alimentation des plages en sable. 
Pour déterminer ce taux de transport, il est nécessaire de calculer le flux à partir de la concentration des sédiments en suspension. En génie côtier, un objectif principal consiste en l'amélioration de la prédiction du transport sédimentaire près du littoral. Beaucoup de chercheurs pensent que pour optimiser la prédiction du transport sédimentaire côtier, il est important $\mathrm{d}^{\prime}$ 'améliorer notre compréhension des processus physiques qui concernent les écoulements en environnement marin.

Dans cet environnement, la houle et les courants sont les principaux phénomènes qui font déplacer les sédiments marins.

Notre problématique consiste à décrire -pour l'ingénieur civil, spécialiste en génie côtier le mélange turbulent avec des outils phénoménologiques (sans hypothèse arbitraire) accessibles en pratique.

\section{Profil vertical de longueur de mélange}

Afin de représenter un courant marin ou fluvial, on considère ici un fluide en écoulement horizontal, unidimensionnel (verticale $\mathrm{z}$ ) et turbulent sur une épaisseur $\mathrm{h}$. Ce paramètre $\mathrm{h}$ peut représenter soit la profondeur totale d'eau si l'écoulement est turbulent dans tout son volume, soit l'épaisseur $\mathrm{m}$ de la couche limite turbulente au fond.

Les sédiments en suspension suivent une loi de concentration de profil $\mathrm{c}(\mathrm{z})$ à déterminer. Ils sont soumis à un flux de sédimentation, descendant, sous l'effet de la gravité, de la poussée d'Archimède et des frottements, mais aussi à un flux net ascendant dû au mouvement des structures turbulentes dans le fluide en écoulement.

Von Kármán a développé la théorie de la longueur de mélange pour la description d'un écoulement turbulent unidirectionnel (x) et unidimensionnel ( $\mathrm{z}$, orienté ici vers le haut) d'un fluide incompressible. Considérant la décomposition usuelle du profil turbulent des vitesses comme la somme $\mathrm{v}=\mathrm{v}+\mathrm{v}^{\prime}$ d'une vitesse moyenne et $\mathrm{d}^{\prime}$ une fluctuation, il a exprimé la longueur de mélange $\mathrm{lm}$ en fonction du profil moyen des vitesses et a obtenu :

$$
l_{m}=-\kappa \cdot \frac{\frac{\partial \overline{\mathbf{v}}}{\partial z}}{\frac{\partial^{2} \overline{\mathbf{v}}}{\partial z^{2}}}
$$

où $\kappa$ est la constante de von Kármán $(\mathrm{x}=0.4)$. Pour cela, il a utilisé l'hypothèse de 2 similitude, qui assure une forme d'invariance d'échelle des caractéristiques (dimension et vitesse) des structures turbulentes.

Par ailleurs, 1'équilibre entre production et dissipation de l'énergie cinétique de la turbulence permet d'établir la proportionnalité $\partial \overline{\mathrm{v}} / \partial z \sim \sqrt{k} / l_{m}$ entre gradient de vitesse moyenne et énergie cinétique de la turbulence (associée aux fluctuations de vitesse) $\sqrt{k}$.

On a donc:

$$
l_{m}=-\kappa \cdot \frac{\frac{\sqrt{k}}{l_{m}}}{\frac{\partial\left(\frac{\sqrt{k}}{l_{m}}\right)}{\partial z}}
$$


Une connaissance du profil vertical de $\sqrt{k}$ permettrait donc d'exprimer à son tour le profil de longueur de mélange $1 \mathrm{~m}^{l_{m}}(z)$.

Plusieursz auteurs proposent une décroissance exponentielle de $\sqrt{k}$ que nous adoptons ici:

$$
\sqrt{k} \sim \mathrm{v}_{f} \cdot \exp \left(-\frac{z}{h}\right)
$$

où $\mathrm{v} f$ est la vitesse de frottement.

Notons dès maintenant que l'expression (3) est particulièrement adaptée à l'étude des sédiments marins ou fluviaux, dont l'accumulation se traduit par la présence d'un fond « mobile », où la vitesse de l'eau ne s'annule pas en limite (on constate que $\sqrt{k}$ ne s'annule ici pour aucune valeur de z). Cette même relation ne permettrait pas de considérer le cas d'un fond fixe.

En introduisant la rugosité physique $\mathrm{r}$ du fond dans notre modèle, et en la définissant comme la valeur moyenne du profil réel (« lissage »), on permet un traitement unidimensionnel (indépendant de $\mathrm{x}$ ) du problème. On a alors bien sûr $\mathrm{r}<\mathrm{z}<\mathrm{h}$ dans le fluide.

Les équations (3) et (2), avec une valeur imposée $\kappa r$ en $r$, fournissent après intégration sur la profondeur d'eau :

$$
l_{m}(z)=\kappa \cdot\left[h-(h-r) \cdot \exp \left(-\frac{(z-r)}{h}\right)\right]
$$

C'est le profil de longueur de mélange que nous choisissons d'adopter ici.

\section{Discussion du profil}

Le profil vertical de longueur de mélange (4), non linéaire, est, comme le montre la figure (1.a), très différent de celui de Prandtl en $l_{m}(z) \approx \kappa \cdot z$.

Pour se rapprocher des valeurs expérimentales (Figure 1.b), d'autres auteurs proposent une hypothèse arbitraire, qui consiste à multiplier la longueur de mélange par $\sqrt{1-(z / h)}$.

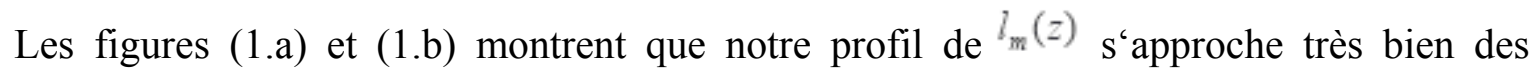
valeurs expérimentales, et ceci sans aucune hypothèse arbitraire. L'écart près de la surface $(\mathrm{z}$ $\approx \mathrm{h}$ ) rend seulement compte de conditions aux limites différentes (surface mobile, et non fixe, dans notre cas).

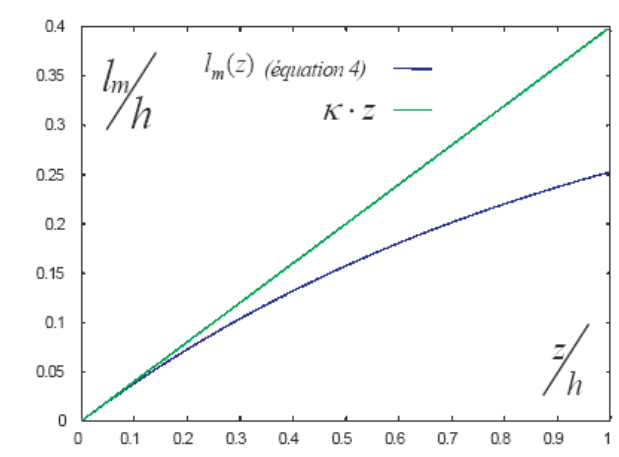

Figure 1.a. Tracé (courbe) du profil théorique ${ }^{l_{m}(z)}$ (donné par l'équation (4). Comparaison avec la droite $\kappa . z$ . On note que ${ }^{l_{m}(z)}$ est partout inférieur à $\kappa . z$. 


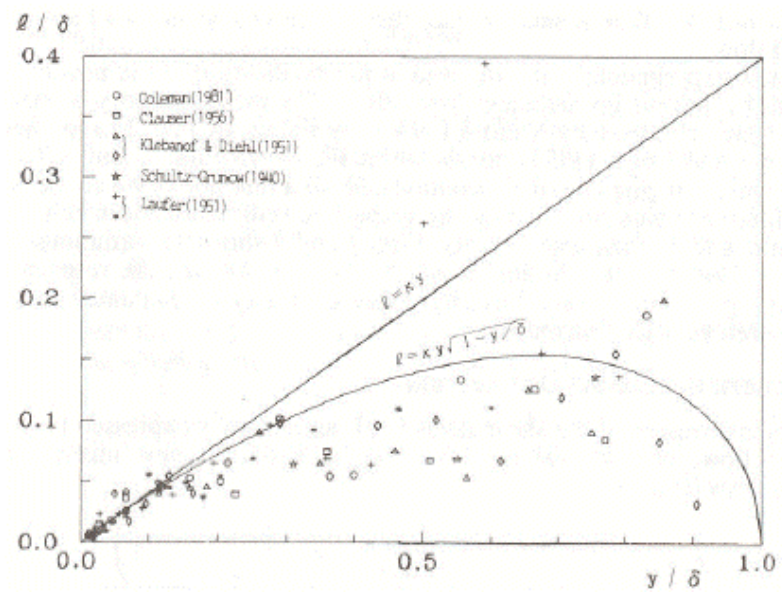

Figure 1.b. Comparaison de la droite $\kappa . z$ avec les mesures. En multipliant la longueur de mélange par $\sqrt{1-(z / h)}$ celle-ci s'approche mieux des valeurs expérimentales (figure: Umeyama et Gerritsen. Ici : $z=y$ et $h==\tilde{O}$ ), dont rend bien compte également le profil (Figure 1.a) de l'équation (4).

On constate que, partant d'une même valeur (imposée) $\kappa . r$ en $r$, notre longueur de mélange croît plus lentement avec z, son gradient dlm/dz de valeur $\kappa \cdot(1-r / h) \cdot \exp (-(z-r) / h)$, étant en tout point (puisque $\mathrm{z} \geq \mathrm{r}$ ) inférieur à $\kappa$. Plus encore, la pente à l'origine (soit en $r$ ), égale à K.(1-r/h), décroît avec la rugosité $r$ (Figure 2), ce qui nous conduit naturellement à introduire un kappa effectif $\kappa^{*}$ tel que

$$
\kappa^{*}=\kappa \cdot(1-r / h)
$$

La différence $\kappa^{*} \neq \kappa$ (Figure 3) traduit le fait que, même au premier ordre (linéarisation sur le paramètre $(\mathrm{z}-\mathrm{r} / \mathrm{)}) \mathrm{h}$ supposé petit, soit près du fond), le profil donné par l'équation (4) est distinct de la loi en $\kappa . Z$. Il s'écrit en effet :

$$
l_{m}(z) \approx \kappa^{*} \cdot z+\kappa \cdot \frac{r^{2}}{h}
$$

Les deux profils ne s'identifient donc, même près du fond, que dans le seul cas des faibles rugosités (fond « lisse »). Là seulement, $\kappa^{*} \approx \kappa$ et $\operatorname{lm}(\mathrm{z}) \approx \kappa . \mathrm{z}$.

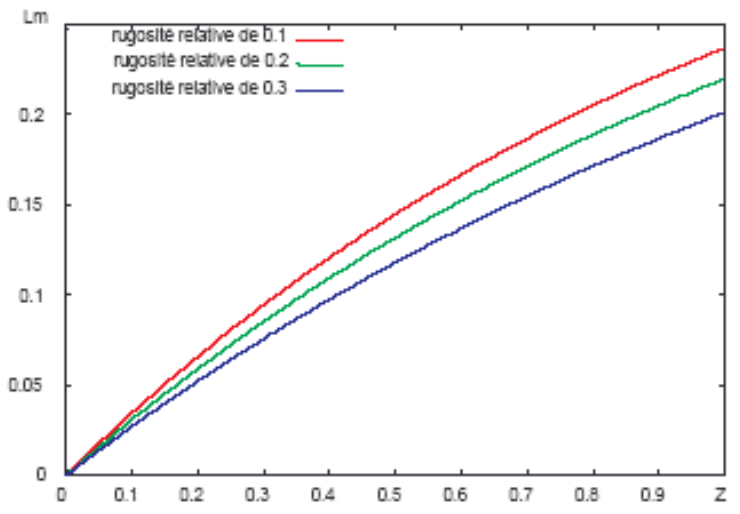

Figure 2. Effet de la rugosité relative $r / h$ sur le profil de lm) ((variation de la longueur de mélange adimensionnelle " $\mathrm{Lm}$ ' en fonction de la distance adimensionnelle $\mathrm{z} / \mathrm{h}$ ). L 'écart à la loi linéaire croît avec $z$, mais aussi avec la rugosité. Trois cas sont représentés, pour trois rugosités. 


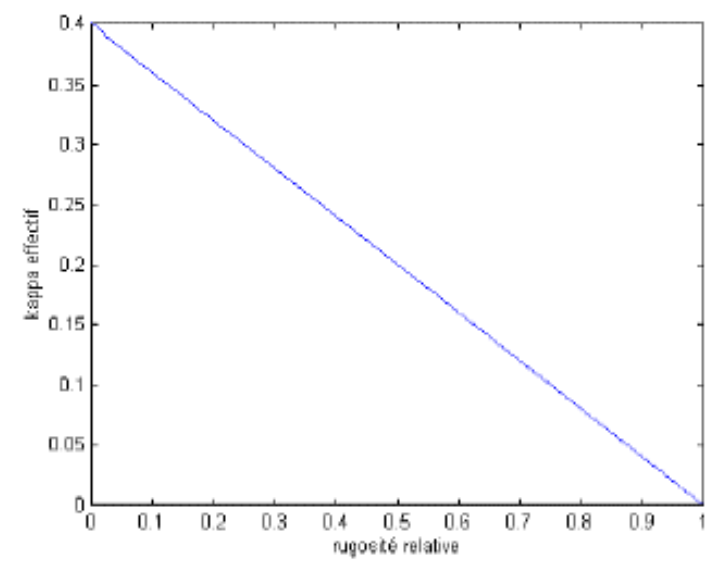

Figure 3. Variation du kappa effectif en fonction de la rugosité relative $r / h$ (pour des fonds rugueux :

$$
\left.\kappa^{*}<\kappa=4,0\right) \text {. }
$$

Nous pouvons conclure que la loi en $\kappa . z$ n'est habilitée à décrire le profil vertical de longueur de mélange qu'au voisinage du fond et (résultat nouveau) dans le cas d'un fond « lisse ».

\section{Ecoulement turbulent en eaux peu profondes - écoulement en couche limite turbulente}

Nous proposons à présent de préciser les conditions d'écoulement effectives, incompatibles avec l'usage d'un profil de longueur de mélange en $\kappa . Z$. L'écart au fond « lisse ") peut surtout se manifester dans le cas d'un écoulement turbulent en eaux peu profondes sur fond très rugueux, ou dans le cas où la couche turbulente se réduit à une couche limite de fond, $\mathrm{d}$ 'épaisseur $\mathrm{m}$ (on a alors $\mathrm{h}=\delta$ ).

Dans ces deux cas, la condition $\mathrm{r} / \mathrm{h}<<1$ peut ne pas être satisfaite et ainsi, comme montrent les équations (5) et (6), le profil $1 \mathrm{~lm}(\mathrm{z})$ peut être notablement différent $\mathrm{m}$ de celui de Prandtl, ceci même près du fond.

Ainsi, c'est essentiellement dans le cas d'un écoulement turbulent en eaux peu profondes ou d'un écoulement en couche limite turbulente que s'impose le plus usage du profil vertical $\operatorname{lm}(\mathrm{z})$ proposé ici.

Notons cependant que, quelle que soit la profondeur d'eau et en présence ou non d'une couche limite turbulente, donc pour toute valeur du rapport rugosité/profondeur (ou rugosité/épaisseur de la couche limite), l'expression (4) de $\operatorname{lm}(\mathrm{z})$ doit remplacer $\kappa . z$ dans la partie supérieure de l'écoulement turbulent $\mathrm{m}$ (condition $\mathrm{z} / \mathrm{h}<<1$ non satisfaite).

Le profil de longueur de mélange que nous proposons ici voit ainsi son usage justifié dans

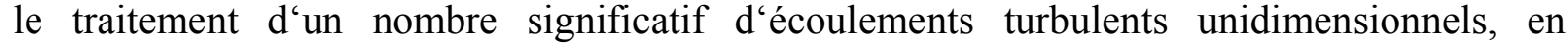
l'absence de conditions aux limites fixes (ce qui est le cas en présence d'un fond sédimentaire, régulier ou non).

\section{$\underline{\text { 5.Profil de concentration sous l'hypothèse des faibles gradients }}$}

L'expression (4) de $\operatorname{lm}(\mathrm{z})$ va nous permettre à présent d'établir l'équation que satisfait la loi de concentration à l'équilibre $\mathrm{c}(\mathrm{z})$ des sédiments. Pour cela, il suffit d'écrire que cet équilibre est réalisé lorsque le flux de sédimentation descendant $\vec{j}_{s}$ compense exactement le flux de mélange ascendant $\vec{j}_{m}$. Le régime permanent js est alors atteint. 
$S^{\prime}$ 'il s'agit d'un écoulement oscillant (cas d'une houle), l'équilibre des flux s'applique alors pour décrire le profil moyen de concentration $\mathrm{c}(\mathrm{z})$, la moyenne temporelle étant effectuée sur une période.

Le flux de sédimentation $\vec{j}_{s}$ est la résultante de l'action sur les sédiments de la s force de gravité, de la poussée d'Archimède et des frottements visqueux. Sur un temps caractéristique très court égal à $\mathrm{m} / \mathrm{K}$, où $\mathrm{m}$ est la masse de la particule solide et $\mathrm{K}$ la constante de frottement (force de frottement en $-K \cdot \overrightarrow{\mathrm{v}}$ ), les sédiments atteignent asymptotiquement une vitesse de sédimentation $\mathrm{v}$ constante, $\mathrm{s}$ proportionnelle à $\mathrm{m}$. Le flux $\vec{j}_{s} \mathrm{~s}$ 'écrit alors en fonction de la concentration, pour js une profondeur $\mathrm{z}$ :

$$
\vec{j}_{s}=c(z) \cdot \overrightarrow{\mathrm{V}}_{\mathrm{s}}
$$

où vs est indépendante de z. L'écriture du flux de mélange $\vec{j}_{m}$ peut être simplement obtenue en appliquant le modèle de la longueur de mélange au cas des faibles gradients de concentration. La linéarisation du problème fournit une proportionnalité entre $\vec{j}_{m}$ et le gradient de concentration, ou loi de Fick, de même $\mathrm{m}$ forme que celle obtenue dans le cas de la diffusion moléculaire :

$$
\vec{j}_{m}=-l_{m} \cdot \mathrm{v}_{m} \cdot \vec{\nabla} c
$$

Ici, $1 \mathrm{~m}$ est la longueur de mélange et vm la vitesse de mélange, fonctions de $\mathrm{z}$. L'hypothèse des faibles gradients $(8) n$ 'est justifiée que dans le cas où $1 \mathrm{~m}$ est très petit devant l'échelle de hauteur de concentration Lc. Nous admettrons cela pour l'instant et proposerons une amélioration (écart à la loi de Fick) en fin d'article, équation (18). L'équilibre des flux impose la relation :

$$
\vec{j}_{s}+\vec{j}_{m}=\overrightarrow{0}
$$

soit, à partir des écritures (7) et (8), et si l'axe vertical Oz est orienté vers le haut :

$$
\frac{\partial c}{\partial z}+\frac{\mathrm{v}_{s}}{l_{m}(z) \cdot \mathrm{v}_{m}(z)} \cdot c(z)=0
$$

Dans le cas (peu réaliste) d'un produit lm.vm supposé constant, la solution de l'équation (10) suit la loi exponentielle :

$$
c(z)=c(r) \cdot \exp \left(-\frac{(z-r)}{L_{c}}\right)
$$

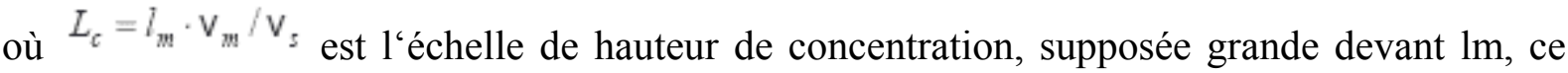
qui n'est justifié que si la vitesse de mélange est très supérieure à la vitesse de sédimentation.

De manière plus réaliste, on peut admettre que le profil spatial de vitesse de mélange suit une décroissance exponentielle 3,7 :

$$
\mathrm{v}_{m}(z)=\mathrm{v}_{m}(r) \cdot \exp \left(-\frac{(z-r)}{L_{\mathrm{v}}}\right)
$$

où Lv définit l'échelle de hauteur de vitesse. On le voit, à ce stade de notre développement, la

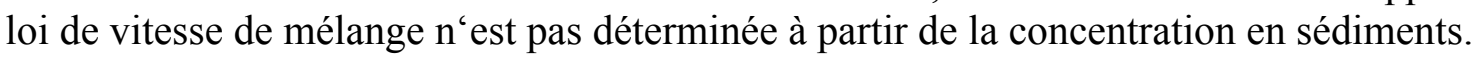


Son expression (12), communément admise, est seulement imposée à partir de résultats $\mathrm{d}^{\prime}$ 'expériences. En ce sens, notre traitement du problème ne constitue pas un couplage complet des deux profils (de concentration et de vitesse, soit des sédiments et du fluide) : le profil c(z) est déduit de la loi de vitesse vm(z).

Le choix courant du profil de longueur de mélange linéaire $l_{m}(z)=\kappa \cdot z$ permettrait de réécrire l'équation (10) sous la forme :

$$
\frac{\partial c}{\partial z}+\frac{\mathrm{v}_{s} \cdot \exp \left(\frac{z-r}{L_{\mathrm{v}}}\right)}{\mathrm{v}_{m}(r) \cdot \kappa \cdot z} \cdot c(z)=0
$$

Nous avons justifié plus haut 1'usage d'une longueur de mélange ayant un profil vertical plus complexe, qui ne tend qu'en limite, en r, vers א.r (4). L“équation (10) s'écrit maintenant:

$$
\frac{\partial c}{\partial z}+\frac{\mathrm{v}_{s} \cdot \exp \left(\frac{z-r}{L_{\mathrm{v}}}\right)}{\mathrm{v}_{m}(r) \cdot \kappa \cdot\left[h-(h-r) \cdot \exp \left(-\frac{(z-r)}{h}\right)\right]} \cdot c(z)=0
$$

où h est la profondeur d'eau, ou la hauteur de la couche limite. Ainsi, la loi de concentration $\mathrm{c}(\mathrm{z})$ est modifiée même près du fond par le choix d'un profil de longueur de mélange donné par (4).

\section{Discussion: gradient de concentration}

Le profil (4) fournit, pour une valeur quelconque de $z$, une longueur de mélange inférieure à la quantité א.Z . Par ailleurs, comme le montre (10), l'échelle de hauteur de concentration s'écrit :

$$
L_{c} \sim \frac{l_{m} \cdot \mathrm{v}_{m}}{\mathrm{v}_{s}}
$$

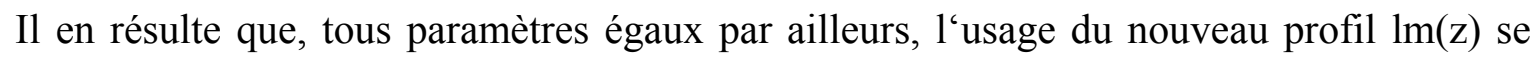
traduit par une diminution de l'échelle de hauteur de concentration Lc, donc par une augmentation du gradient de concentration. Le profil de concentration est donc plus «marqué » que sous l'hypothèse d'une longueur de mélange en $\kappa . z$ : à l'équilibre, les sédiments sont plus massivement concentrés vers le fond.

Ce résultat, fondamental, est confirmé par de nombreux résultats de mesures . Cette tendance constatée à la prévision de gradients de concentrations sédimentaires plus importants nous oblige maintenant, avant de conclure, à envisager une voie d'amélioration future de notre étude, à l'usage d'une application aux situations de très forts gradients.

\section{Cas des forts gradients de concentration}

Comme nous 1'avons dit, la loi de Fick (8) pour l'écriture du flux de mélange n'est justifiée que sous l'hypothèse des faibles gradients de concentration ; plus précisément, si un

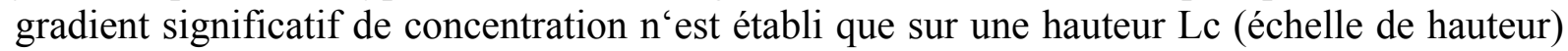
très grande devant $\mathrm{lm}$. Nous avons jusqu'à présent admis cette condition. Si elle n'est pas 
satisfaite (forts gradients), une modification de l'écriture du flux de mélange donc de (14) $s$ 'impose.

Le modèle de la longueur de mélange entraîne que le flux net de mélange s'écrit comme le bilan d'une " montée » des sédiments situés à la base d'une structure (hauteur $z-1 \mathrm{~m} / 2$ ) et d'une " descende » de ceux placés à son sommet (hauteur $z+1 \mathrm{~m} / 2$ ). En projection sur l'axe Oz orienté vers le haut, on obtient alors l'écriture $m$ du flux de mélange :

$$
j_{m}=\mathrm{v}_{m} \cdot\left(c\left(z-l_{m} / 2\right)-c\left(z+l_{m} / 2\right)\right)
$$

Cette expression ne redonne la loi de Fick (8) qu'après linéarisation sur le paramètre $1 \mathrm{~m}$, supposé très petit devant Lc, soit sous 1'hypothèse d'un faible gradient de concentration. Alors, en adoptant notre profil de longueur de mélange (4), on obtient (14). Dans le cas contraire (forts gradients), et sous la condition que le gradient de concentration ne soit pas trop important (condition $1 \mathrm{~m}<\mathrm{lc}$ satisfaite), on peut admettre un développement de (16) à 1'ordre 3 sur $1 \mathrm{~m}$ ( $1^{\prime}$ ordre 2 ne produisant aucun terme supplémentaire), soit :

$$
j_{m} \approx-l_{m} \cdot v_{m} \cdot \frac{\partial c}{\partial z}-\frac{l_{m}^{3} \cdot v_{m}}{24} \cdot \frac{\partial^{3} c}{\partial z^{3}}
$$

Et donc, à l'équilibre (voir aussi) :

$$
\frac{l_{m}^{2}}{24} \cdot \frac{\partial^{3} c}{\partial z^{3}}+\frac{\partial c}{\partial z}+\frac{\mathrm{v}_{s}}{l_{m}(z) \cdot \mathrm{v}_{m}(z)} \cdot c(z)=0
$$

au lieu de (10), où la dérivée d'ordre 3 est bien sûr nulle. L'introduction, dans le cas des forts gradients, de la longueur de mélange (4) dans (18), devra permettre d'améliorer notre modèle pour la description des profils de concentrations sédimentaires très marqués. Notons déjà que, pour un profil de concentration usuel (exponentiel), la dérivée d'ordre 3 dans (18) est négative, d'où un effet de diminution (en valeur absolue) du gradient $\delta c / \delta z$.

Ainsi, alors que la nouvelle longueur de mélange (4) tend à accroître le gradient de concentration sédimentaire, l'écart à la loi de Fick (forts gradients) a un effet opposé.

Il est par ailleurs aisé de montrer que ce dernier effet est d'autant plus important que le rapport $\mathrm{Vs} / \mathrm{Vm}$ est grand, c'est-à-dire pour les gros sédiments, dont la vitesse de sédimentation vs est plus importante.

On peut donc prévoir, par l'effet conjugué du profil (4) de lm et de la prise en compte (17) des forts gradients, une augmentation (par comparaison aux prévisions " classiques ») du gradient de concentration sédimentaire dans le cas des petits sédiments (rôle de (4) dominant), et une diminution dans le cas contraire (rôle de (17) dominant). Là encore, des résultats de mesures tendent à le confirmer. Peut-être déterminera-t-on, à l'équilibre des deux processus, une dimension « critique » des sédiments, pour laquelle on rejoindra les prévisions « classiques $\gg . .$.

\section{Conclusion}

Dans cette communication, nous avons introduit une écriture du profil vertical $\operatorname{lm}(\mathrm{z}) \mathrm{de}$ longueur de mélange plus complète que $1^{\prime}$ expression usuelle en $1 \mathrm{~m}=\kappa \cdot \mathrm{Z}$.

Nous avons montré que notre expression de $1 \mathrm{~m}$ ne tend en limite vers celle de Prandtl que dans le seul cas des faibles rugosités et près du fond. Nous avons ainsi proposé une alternative à l'usage de la forme $1 \mathrm{~m}=\kappa . z$ pour la description d'un $\mathrm{m}$ écoulement turbulent en eaux peu profondes ou pour celle d'un écoulement en couche limite turbulente. 
Puis sous 1'hypothèse (discutée) de validité de la loi de Fick pour l'écriture du flux de mélange, nous avons établi la nouvelle équation portant sur le profil vertical de concentration $\mathrm{c}(\mathrm{z})$ des sédiments.

La comparaison avec les prévisions théoriques usuelles a permis d'expliquer très simplement certains écarts théorie/expérience constatés. Plus particulièrement, notre modèle prévoit, pour chaque profondeur, une longueur de mélange plus petite, d'où un gradient de concentration plus important, résultat compatible avec les données expérimentales, ceci sans faire intervenir d'autre hypothèse arbitraire.

Enfin, une critique a été faite de l'usage de la loi de Fick pour l'écriture du flux de mélange dans le cas des forts gradients de concentration. Dans ce cas, on prévoit une diminution du gradient $\mathrm{c}(\mathrm{z})$, en accord avec les données expérimentales portant sur les gros sédiments.

\section{Références}

1 Von Kármán Th. (1930). Mechanische Aehnlichkeit und turbulenz. Nach. Ges. Wiss. Göttingen, Math. Phys. Klasse, 58.

2 Schlichting H. (1968). Boundary layer theory. Mc Graw-hill book company, New York.

3 Nezu I. and Nakagawa H. (1993). Turbulence in open-channel flows. A.A. Balkema, Rotterdam.

4 Absi R. (2002). Wave boundary layer instability near flow reversal. Proc. 28th International Coastal Engineering Conf. ASCE, 532-544. World Scientific.

5 Nezu I. and Rodi W. (1986). Open-channel flow measurements with a laser Doppler anemometer. J. Hydraul. Eng., 112(5), 335oe355.

6 Umeyama M. and Gerritsen F. (1992). Velocity distribution in uniform sediment-laden flow. J. Hydraulic Engineering, 118 (2), 229-245.

7 Nielsen P. and Teakle A.-L. (submitted to -Physics of Fluids"). Turbulent diffusion of momentum and suspended particles : a finite-mixing-length-theory.

8 Dohmen-Janssen C.M., Hassan W.N. and Ribberink J.S. (2001). Mobile-bed effects in oscillatory sheet flow. Journal of Geophysical Research, 106, 27,103-27,115. 